\title{
Polynomial integrals for third- and fourth-order ordinary difference equations
}

\author{
$R$ Sahadevan ${ }^{a}$ and $C$ Uma Maheswari ${ }^{b}$ \\ Ramanujan Institute for Advanced Study in Mathematics, University of Madras, \\ Chennai-600 005, Tamil Nadu, India. \\ ${ }^{a}$ E-mail: ramajayamsaha@yahoo.co.in \\ ${ }^{b}$ E-mail: umagenie2004@hotmail.com
}

Received May 15, 2008; Accepted September 1, 2008

\begin{abstract}
A direct method to construct polynomial integrals for third order ordinary difference equation $(O \Delta E) w(n+3)=F(w(n), w(n+1), w(n+2))$ and fourth order $O \Delta E$ $w(n+4)=F(w(n), w(n+1), w(n+2), w(n+3))$ is presented. The effectiveness of the method to construct more than one polynomial integral for $\mathrm{N}$-th order $O \Delta E$ is also briefly discussed.
\end{abstract}

\section{Introduction}

The discrete nonlinear systems governed by both ordinary difference equations or mappings and partial difference equations or lattice equations have drawn much attention by researchers working under different areas of applied science $[1,2,3,5,7,9,10]$. Since discrete systems governed by difference equations are more fundamental than the continuous ones described by differential equations their study becomes essential which will lead to the development of a general theory of discrete and in particular nonlinear difference equations. Even though there exists no unique definition of integrability considerable number of analytical methods have been formulated by different groups in recent years to deal with integrability $[4,7,8,10,11,12,13,16,18,19,20,21,22,24,25]$ and significant advancement has already been made for the second order both for autonomous and nonautonomous cases $[5,7,9,10,17,19,20,25]$. We take the working definition of integrability, here, the one which is related with the existence of sufficient number of integrals of an $O \Delta E$. An integral (also referred to as conserved quantity) of $O \Delta E$ is a function that is not identically constant but is constant on all solutions of it. An autonomous N-th order nonlinear $O \Delta E$ is said to be integrable if it admits (N-1) functionally independent integrals. Note that if a difference equation is measure preserving in dimension $N$ and has (at least) $(N-2)$ independent integrals then it has a (degenerate) Poisson structure, so defines a symplectic map on each 2D level set of these integrals [6]. Given an autonomous $\mathrm{N}$-th order nonlinear $O \Delta E$ there exists no systematic analytic technique to derive its integrals enabling one to investigate its integrability. Recently a direct method was proposed 
for $N$-th order autonomous difference equation to construct rational integrals and several new integrable difference equations of higher order were identified [23]. In this article we present a method to construct polynomial integrals through third and fourth order $O \Delta E$ [ see 14,15 for a different method]. The effectiveness of the method is also discussed for $N$ -th order $O \Delta E$.

The plan of the article is as follows. In $\S 2$ we describe the method, which involves factorisation, through third order autonomous $O \Delta E w_{n+3}=F\left(w_{n}, w_{n+1}, w_{n+2}\right), w_{n}=$ $w(n), w_{n+1}=w(n+1)$ etc. to derive 2 polynomial integrals. In $\S 3$ we extend it for fourthorder autonomous $O \Delta E w_{n+4}=F\left(w_{n}, w_{n+1}, w_{n+2}, w_{n+3}\right)$ and identify $F$ admitting 2 independent integrals. In $\S 4$ we present a brief summary of our results. The effectiveness of the method is also discussed for $N$-th order $O \Delta E$ in the Appendix.

\section{Construction of integrals for third-order autonomous dif- ference equation}

Consider an autonomous third order $O \Delta E$ having the form

$$
w_{n+3}=F\left(w_{n}, w_{n+1}, w_{n+2}\right) \text { or } w_{3}=F\left(w_{0}, w_{1}, w_{2}\right) .
$$

Hereafter, we denote $w_{0}=w_{n}, w_{1}=w_{n+1}, \ldots, w_{N}=w_{n+N}$ unless otherwise specified. Assume that equation (2.1) admits an integral $I\left(w_{0}, w_{1}, w_{2}\right)$ having the form

$$
I\left(w_{0}, w_{1}, w_{2}\right)=\sum_{j=1}^{3}\left[A_{1 j}\left(w_{1}\right) w_{2}^{2}+A_{2 j}\left(w_{1}\right) w_{2}+A_{3 j}\left(w_{1}\right)\right] w_{0}^{3-j} .
$$

The integrability condition $I\left(w_{0}, w_{1}, w_{2}\right)-I\left(w_{1}, w_{2}, w_{3}\right)=0$ leads to a quadratic equation in $w_{3}$

$$
F_{1}\left(w_{1}, w_{2}\right) w_{3}^{2}+F_{2}\left(w_{1}, w_{2}\right) w_{3}-\left[F_{4}\left(w_{1}, w_{2}\right) w_{0}^{2}+F_{5}\left(w_{1}, w_{2}\right) w_{0}+F_{6}\left(w_{1}, w_{2}\right)-F_{3}\left(w_{1}, w_{2}\right)\right]=0
$$

where

$$
\begin{aligned}
& F_{i}\left(w_{1}, w_{2}\right)=\sum_{j=1}^{3} A_{i j}\left(w_{2}\right) w_{1}^{3-j}, i=1,2,3 \\
& F_{k+3}\left(w_{1}, w_{2}\right)=\sum_{j=1}^{3} A_{j k}\left(w_{1}\right) w_{2}^{3-j}, k=1,2,3 .
\end{aligned}
$$

Hereafter we denote $F_{i}\left(w_{1}, w_{2}\right)$ as $F_{i}$ for the remaining section unless otherwise specified. Equation (2.3) can be factorised as

$$
\left(w_{3}+w_{0}+\frac{F_{5}+F_{2}}{2 F_{1}}\right)\left(w_{3}-w_{0}+\frac{F_{2}-F_{5}}{2 F_{1}}\right)=0
$$


provided

$$
A_{11}\left(w_{2}\right) w_{1}^{2}+A_{12}\left(w_{2}\right) w_{1}+A_{13}\left(w_{2}\right)=A_{11}\left(w_{1}\right) w_{2}^{2}+A_{21}\left(w_{1}\right) w_{2}+A_{31}\left(w_{1}\right)
$$

and

$$
\left(F_{5}+F_{2}\right)\left(F_{5}-F_{2}\right)=4\left(F_{6}-F_{3}\right) F_{1}
$$

Thus we obtain,

$$
\begin{aligned}
& w_{3}=-w_{0}-\frac{F_{2}+F_{5}}{2 F_{1}}, \\
& w_{3}=w_{0}-\frac{F_{2}-F_{5}}{2 F_{1}} .
\end{aligned}
$$

Solving equation $(2.5 \mathrm{a})$ yields

$$
\begin{aligned}
& A_{11}\left(w_{2}\right)=a_{1} w_{2}^{2}+a_{2} w_{2}+a_{3}, \\
& A_{21}\left(w_{2}\right)=a_{2} w_{2}^{2}+b_{2} w_{2}+c_{2}, \\
& A_{31}\left(w_{2}\right)=a_{3} w_{2}^{2}+b_{3} w_{2}+c_{3}, \\
& A_{12}\left(w_{2}\right)=a_{2} w_{2}^{2}+b_{2} w_{2}+b_{3}, \\
& A_{13}\left(w_{2}\right)=a_{3} w_{2}^{2}+c_{2} w_{2}+c_{3},
\end{aligned}
$$

where $a_{1}, a_{2}, a_{3}, b_{2}, b_{3}, c_{2}$ and $c_{3}$ are arbitrary constants. Equation $(2.5 \mathrm{~b})$ suggests that it can be solved for two distinct possibilities:

(i) $\quad F_{5}=F_{2}$ and $\quad F_{6}=F_{3}$,

(ii) $\quad F_{5} \neq F_{2}$ and $F_{6} \neq F_{3}$.

The conditions given in equation (2.9) can be rewritten respectively as

$$
\begin{aligned}
& A_{12}\left(w_{1}\right) w_{2}^{2}-A_{21}\left(w_{2}\right) w_{1}^{2}=A_{22}\left(w_{2}\right) w_{1}-A_{22}\left(w_{1}\right) w_{2}+A_{23}\left(w_{2}\right)-A_{32}\left(w_{1}\right), \\
& A_{13}\left(w_{1}\right) w_{2}^{2}-A_{31}\left(w_{2}\right) w_{1}^{2}=A_{32}\left(w_{2}\right) w_{1}-A_{23}\left(w_{1}\right) w_{2}+A_{33}\left(w_{2}\right)-A_{33}\left(w_{1}\right) .
\end{aligned}
$$

Making use of the forms for $A_{11}\left(w_{2}\right), A_{12}\left(w_{2}\right), A_{13}\left(w_{2}\right), A_{21}\left(w_{2}\right)$ and $A_{31}\left(w_{2}\right)$ given in equations (2.8a)-(2.8e) in (2.11) and (2.12) we obtain

$$
\begin{aligned}
& A_{22}\left(w_{2}\right)=b_{2} w_{2}^{2}+e_{2} w_{2}+e_{3}, \\
& A_{23}\left(w_{2}\right)=b_{3} w_{2}^{2}+e_{3} w_{2}+f_{3}, \\
& A_{32}\left(w_{2}\right)=c_{2} w_{2}^{2}+e_{3} w_{2}+f_{3}, \\
& A_{33}\left(w_{2}\right)=c_{3} w_{2}^{2}+f_{3} w_{2}+j_{3},
\end{aligned}
$$

where $e_{2}, e_{3}, f_{3}$ and $j_{3}$ are arbitrary constants. Thus we obtain a third order difference equation

$w_{3}=-w_{0}-\frac{\left(a_{2} w_{2}^{2}+b_{2} w_{2}+c_{2}\right) w_{1}^{2}+b_{2} w_{1} w_{2}^{2}+e_{2} w_{1} w_{2}+e_{3} w_{1}+b_{3} w_{2}^{2}+e_{3} w_{2}+f_{3}}{\left(a_{1} w_{2}^{2}+a_{2} w_{2}+a_{3}\right) w_{1}^{2}+a_{2} w_{1} w_{2}^{2}+b_{2} w_{1} w_{2}+b_{3} w_{1}+a_{3} w_{2}^{2}+c_{2} w_{2}+c_{3}}$ 
with one integral

$$
\begin{aligned}
& I_{1}=\left[\left(a_{1} w_{1}^{2}+a_{2} w_{1}+a_{3}\right) w_{0}^{2}+\left(a_{2} w_{1}^{2}+b_{2} w_{1}+b_{3}\right) w_{0}+a_{3} w_{1}^{2}+c_{2} w_{1}+c_{3}\right] w_{2}^{2} \\
& \quad+\left[\left(a_{2} w_{1}^{2}+b_{2} w_{1}+c_{2}\right) w_{0}^{2}+\left(b_{2} w_{1}^{2}+e_{2} w_{1}+e_{3}\right) w_{0}+b_{3} w_{1}^{2}+e_{3} w_{1}+f_{3}\right] w_{2} \\
& \quad+\left(a_{3} w_{1}^{2}+b_{3} w_{1}+c_{3}\right) w_{0}^{2}+\left(c_{2} w_{1}^{2}+e_{3} w_{1}+f_{3}\right) w_{0}+c_{3} w_{1}^{2}+f_{3} w_{1} .
\end{aligned}
$$

We would like to mention that equation (2.14) can also be rewritten as

$$
w_{3}=-w_{0}-\frac{\left(\alpha_{1} w_{1} w_{2}+\alpha_{2} w_{1}+\alpha_{3} w_{2}+\alpha_{4}\right)\left(\gamma_{1} w_{1} w_{2}+\gamma_{2} w_{1}+\gamma_{3} w_{2}+\gamma_{4}\right)}{\left(\alpha_{1} w_{1} w_{2}+\alpha_{2} w_{1}+\alpha_{3} w_{2}+\alpha_{4}\right)\left(\beta_{1} w_{1} w_{2}+\beta_{2} w_{1}+\beta_{3} w_{2}+\beta_{4}\right)}
$$

by choosing the constants $a_{1}, a_{2}, a_{3}, b_{2}, b_{3}, c_{2}, c_{3}, e_{3}$ and $f_{3}$ appropriately. For example, equation (2.14) becomes

$$
w_{3}=-w_{0}-\frac{2 \beta_{3} w_{1} w_{2}+\gamma_{2}\left(w_{1}+w_{2}\right)+\gamma_{4}}{\beta_{1} w_{1} w_{2}+\beta_{3}\left(w_{1}+w_{2}\right)+\gamma_{2}-\alpha_{4} \beta_{3}}
$$

provided

$$
\begin{aligned}
& a_{1}=\beta_{1}^{2}, a_{2}=2 \beta_{1} \beta_{3}, a_{3}=\beta_{3}^{2}, \\
& b_{2}=\left(\beta_{1} \gamma_{2}+2 \beta_{3}^{2}\right), c_{2}=b_{3}=\gamma_{2} \beta_{3}, c_{3}=\alpha_{4} \beta_{3}\left(\gamma_{2}-\alpha_{4} \beta_{3}\right), \\
& e_{2}=\left(2 \gamma_{2} \beta_{3}+\beta_{1} \gamma_{4}+2 \alpha_{4} \beta_{3}^{2}\right), e_{3}=\beta_{3}\left(\alpha_{4} \gamma_{2}+\gamma_{4}\right), f_{3}=\alpha_{4} \gamma_{4} \beta_{3}, j_{3} \text {-arbitrary }
\end{aligned}
$$

and the integral (2.15) becomes

$$
\begin{aligned}
& I_{1}=\left(\beta_{1} w_{0} w_{1}+\beta_{3}\left(w_{0}+w_{1}\right)+\alpha_{4} \beta_{3}\right)\left[\left(\beta_{1} w_{0} w_{1}+\beta_{3}\left(w_{0}+w_{1}\right)+\gamma_{2}-\alpha_{4} \beta_{3}\right) w_{2}\right. \\
& \left.\quad+2 \beta_{3} w_{0} w_{1}+\gamma_{2}\left(w_{0}+w_{1}\right)+\gamma_{4}\right] w_{2}+\beta_{3}\left(w_{1}+\alpha_{4}\right)\left(\beta_{3} w_{0} w_{1}+\gamma_{2} w_{1}\right. \\
& \left.\quad+\left(\gamma_{2}-\alpha_{4} \beta_{3}\right) w_{0}+\gamma_{4}\right) w_{0}+\alpha_{4} \beta_{3}\left(\gamma_{2}-\beta_{3} \alpha_{4}\right) w_{1}^{2}+\alpha_{4} \beta_{3} \gamma_{4} w_{1} .
\end{aligned}
$$

In order to construct second integral $I_{2}$ we use the other possibility given in equation (2.10). It is clear from equation $\left(2.5\right.$ a) that $A_{11}\left(w_{1}\right), A_{12}\left(w_{1}\right), A_{13}\left(w_{1}\right), A_{21}\left(w_{1}\right)$ and $A_{31}\left(w_{1}\right)$ are quadratic polynomials. However, equation (2.5b) suggests that $A_{22}\left(w_{1}\right), A_{23}\left(w_{1}\right)$, $A_{32}\left(w_{1}\right)$ and $A_{33}\left(w_{1}\right)$ may be quartic polynomials. Thus we consider

$$
\begin{aligned}
& A_{11}\left(w_{1}\right)=\tilde{a}_{1} w_{1}^{2}+\tilde{a}_{2} w_{1}+\tilde{a}_{3}, \\
& A_{21}\left(w_{1}\right)=\tilde{a}_{2} w_{1}^{2}+\tilde{b}_{2} w_{1}+\tilde{c}_{2}, \\
& A_{31}\left(w_{1}\right)=\tilde{a}_{3} w_{1}^{2}+\tilde{b}_{3} w_{1}+\tilde{c}_{3}, \\
& A_{12}\left(w_{1}\right)=\tilde{a}_{2} w_{1}^{2}+\tilde{b}_{2} w_{1}+\tilde{b}_{3}, \\
& A_{13}\left(w_{1}\right)=\tilde{a}_{3} w_{1}^{2}+\tilde{c}_{2} w_{1}+\tilde{c}_{3}, \\
& A_{22}\left(w_{1}\right)=\epsilon_{1} w_{1}^{4}+\epsilon_{2} w_{1}^{3}+\epsilon_{3} w_{1}^{2}+\epsilon_{4} w_{1}+\epsilon_{5}, \\
& A_{23}\left(w_{1}\right)=\epsilon_{6} w_{1}^{4}+\epsilon_{7} w_{1}^{3}+\epsilon_{8} w_{1}^{2}+\epsilon_{9} w_{1}+\epsilon_{10}, \\
& A_{32}\left(w_{1}\right)=\epsilon_{11} w_{1}^{4}+\epsilon_{12} w_{1}^{3}+\epsilon_{13} w_{1}^{2}+\epsilon_{14} w_{1}+\epsilon_{15}, \\
& A_{33}\left(w_{1}\right)=\epsilon_{16} w_{1}^{4}+\epsilon_{17} w_{1}^{3}+\epsilon_{18} w_{1}^{2}+\epsilon_{19} w_{1}+\epsilon_{20},
\end{aligned}
$$


where $\tilde{a}_{1}, \tilde{a}_{2}, \tilde{a}_{3}, \tilde{b}_{2}, \tilde{b}_{3}, \tilde{c}_{2}, \tilde{c}_{3}$ and $\epsilon_{i}, i=1,2 \ldots 20$ are unknown constants to be determined. Then equation (2.6) reads

$$
w_{3}=-w_{0}-\frac{\tilde{F}}{2 \tilde{G}}
$$

where,

$$
\begin{aligned}
\tilde{F} & =\epsilon_{11} w_{1}^{4}+\epsilon_{6} w_{2}^{4}+\epsilon_{12} w_{1}^{3}+\epsilon_{7} w_{2}^{3}+\epsilon_{1}\left(w_{1}^{3}+w_{2}^{3}\right) w_{1} w_{2}+\left(\epsilon_{3}+b_{2}\right)\left(w_{1}+w_{2}\right) w_{1} w_{2} \\
& +\epsilon_{2}\left(w_{1}^{2}+w_{2}^{2}\right) w_{1} w_{2}+2 a_{2} w_{2}^{2} w_{1}^{2}+\left(\epsilon_{13}+c_{2}\right) w_{1}^{2}+\left(b_{3}+\epsilon_{8}\right) w_{2}^{2}+2 \epsilon_{4} w_{2} w_{1} \\
& +\left(\epsilon_{5}+\epsilon_{14}\right) w_{1}+\left(\epsilon_{5}+\epsilon_{9}\right) w_{2}+\epsilon_{10}+\epsilon_{15} \\
\tilde{G} & =\left(\tilde{a}_{1} w_{1}^{2} w_{2}^{2}+\tilde{a}_{2} w_{2} w_{1}^{2}+\tilde{a}_{3} w_{1}^{2}+\tilde{a}_{2} w_{1} w_{2}^{2}+\tilde{b}_{2} w_{1} w_{2}+\tilde{b}_{3} w_{1}+\tilde{a}_{3} w_{2}+\tilde{c}_{2} w_{2}+\tilde{c}_{3}\right) .
\end{aligned}
$$

We would like to mention here that both $\tilde{F}$ and $\tilde{G}$ can be factored with a common factor by choosing the constants $\tilde{a}_{1}, \tilde{a}_{2}, \tilde{a}_{3}, \tilde{b}_{2}, \tilde{b}_{3}, \tilde{c}_{2}, \tilde{c}_{3}$ and $\epsilon_{i}, i=1,2 \ldots 20$, appropriately. That is,

$$
\begin{aligned}
& \tilde{F}=\left(\tilde{\alpha}_{1} w_{1} w_{2}+\tilde{\alpha}_{2} w_{1}+\tilde{\alpha}_{3} w_{2}+\tilde{\alpha}_{4}\right)\left[\tilde{\gamma}_{1} w_{1}^{2} w_{2}^{2}+\tilde{\gamma}_{2} w_{1}^{2} w_{2}+\tilde{\gamma}_{3} w_{1} w_{2}^{2}+\tilde{\gamma}_{4} w_{1}^{2}+\tilde{\gamma}_{5} w_{2}^{2}\right. \\
& \left.\quad+\tilde{\gamma}_{6} w_{1} w_{2}+\tilde{\gamma}_{7} w_{1}+\tilde{\gamma}_{8} w_{2}+\tilde{\gamma}_{9}\right] \\
& \tilde{G}=\left(\tilde{\alpha}_{1} w_{1} w_{2}+\tilde{\alpha}_{2} w_{1}+\tilde{\alpha}_{3} w_{2}+\tilde{\alpha}_{4}\right)\left(\tilde{\beta}_{1} w_{1} w_{2}+\tilde{\beta}_{2} w_{1}+\tilde{\beta}_{3} w_{2}+\tilde{\beta}_{4}\right)
\end{aligned}
$$

For example, equation (2.20) reduces into

$$
w_{3}=-w_{0}-\frac{\tilde{\alpha}_{4}\left(4 \tilde{\beta}_{2} w_{1} w_{2}+\tilde{\gamma}_{8}\left(w_{1}+w_{2}\right)+\tilde{\gamma}_{9}\right)}{2 \tilde{\beta}_{1} \tilde{\alpha}_{4} w_{1} w_{2}+2 \tilde{\alpha}_{4} \tilde{\beta}_{2}\left(w_{1}+w_{2}\right)+\tilde{\alpha}_{4} \tilde{\gamma}_{8}-2 \epsilon_{9}}
$$

provided

$$
\begin{aligned}
& \tilde{a_{1}}=\tilde{a_{2}}=\tilde{a_{3}}=0, \tilde{b}_{2}=\tilde{\alpha}_{4} \tilde{\beta}_{1}, \tilde{c}_{2}=\tilde{b}_{3}=\tilde{\alpha}_{4} \tilde{\beta}_{2}, \tilde{c}_{3}=\frac{\tilde{\alpha}_{4} \tilde{\gamma}_{8}}{2}-\epsilon_{9}, \\
& \epsilon_{3}=-\tilde{b}_{2}, \epsilon_{4}=2 \tilde{b}_{3}, \epsilon_{5}=-\epsilon_{9}+\tilde{\alpha}_{4} \tilde{\gamma}_{8}, \epsilon_{8}=-\tilde{b}_{3}, \epsilon_{9}-\text { arbitrary, } \\
& \epsilon_{10}=\epsilon_{15}=\frac{\tilde{\alpha}_{4} \tilde{\gamma}_{9}}{2}, \epsilon_{13}=-\tilde{b}_{3}, \epsilon_{14}=\epsilon_{9}, \epsilon_{18}=-\epsilon_{9}, \\
& \epsilon_{1}=\epsilon_{2}=\epsilon_{6}=\epsilon_{7}=\epsilon_{11}=\epsilon_{12}=\epsilon_{16}=\epsilon_{17}=\epsilon_{19}=\epsilon_{20}=0 .
\end{aligned}
$$

Also the conditions $(2.5 \mathrm{a}, \mathrm{b})$ and (2.10) are identically satisfied for the above parametric restrictions which in turn leads to the existence of second integral $I_{2}$. Furthermore equation (2.21) can be rewritten as

$$
w_{n+3}=-w_{n}-\frac{2 \lambda_{1} w_{n+1} w_{n+2}+\lambda_{2}\left(w_{n+1}+w_{n+2}\right)+\lambda_{3}}{\lambda_{4} w_{n+1} w_{n+2}+\lambda_{1}\left(w_{n+1}+w_{n+2}\right)+\lambda_{5}}
$$

where

$$
\tilde{\beta}_{2}=\frac{\lambda_{1}}{2 \tilde{\alpha}_{4}}, \tilde{\gamma}_{8}=\frac{\lambda_{2}}{\tilde{\alpha}_{4}}, \tilde{\gamma}_{9}=\frac{\lambda_{3}}{\tilde{\alpha}_{4}}, \tilde{\beta}_{1}=\frac{\lambda_{4}}{2 \tilde{\alpha}_{4}}, \epsilon_{9}=\frac{\alpha_{4} \gamma_{8}-\lambda_{5}}{2}
$$


and so the integral $I_{2}$ is

$$
\begin{aligned}
I_{2} & =\left[\lambda_{4} w_{n+1} w_{n}+\lambda_{1} w_{n}+\lambda_{1} w_{n+1}+\lambda_{5}\right] w_{n+2}^{2}+\left[\lambda_{4} w_{n+1} w_{n}^{2}+\lambda_{1} w_{n}^{2}-\lambda_{4} w_{n+1}^{2} w_{n}\right. \\
& \left.+2 \lambda_{1} w_{n} w_{n+1}+\lambda_{2} w_{n}+\lambda_{5} w_{n}-\lambda_{1} w_{n+1}^{2}+\left(\lambda_{2}-\lambda_{5}\right) w_{n+1}+\lambda_{3}\right] w_{n+2}+\lambda_{1} w_{n+1} w_{n}^{2} \\
& +\lambda_{5} w_{n}^{2}-\lambda_{1} w_{n+1}^{2} w_{n}+\left(\lambda_{2}-\lambda_{5}\right) w_{n+1} w_{n}+\lambda_{3} w_{n}+\left(\lambda_{5}-\lambda_{2}\right) w_{n+1}^{2} .
\end{aligned}
$$

It is easy to see that the third order $O \Delta E$ (2.17) admitting the integral $I_{1}$ assumes exactly the same form as (2.22) by choosing the parameters $\beta_{1}, \beta_{3}, \gamma_{2}$ and $\gamma_{4}$ as

$$
\alpha_{4}=\frac{\lambda_{2}-\lambda_{5}}{\lambda_{1}}, \beta_{1}=\lambda_{4}, \beta_{3}=\lambda_{1}, \gamma_{2}=\lambda_{2}, \gamma_{4}=\lambda_{3}
$$

and the integral $I_{1}$ becomes

$$
\begin{aligned}
& I_{1}=\left(\lambda_{4} w_{n} w_{n+1}+\lambda_{1} w_{n}+\lambda_{1} w_{n+1}+\lambda_{2}-\lambda_{5}\right)\left[\left(\lambda_{4} w_{n} w_{n+1}+\lambda_{1} w_{n}+\lambda_{1} w_{n+1}+\lambda_{5}\right) w_{n+2}\right. \\
& \left.\quad+2 \lambda_{1} w_{n} w_{n+1}+\lambda_{2} w_{n}+\lambda_{2} w_{n+1}+\lambda_{3}\right] w_{n+2}+\left(\lambda_{1} w_{n+1}+\lambda_{2}-\lambda_{5}\right)\left[\lambda_{1} w_{n} w_{n+1}+\lambda_{5} w_{n}\right. \\
& \left.\quad+\lambda_{2} w_{n+1}+\lambda_{3}\right] w_{n}+\left(\lambda_{5} w_{n+1}+\lambda_{3}\right)\left(\lambda_{2}-\lambda_{5}\right) w_{n+1} .
\end{aligned}
$$

Thus we conclude that the third order $O \Delta E$ (2.22) admits 2 independent integrals $I_{1}$ and $I_{2}$.

Proceeding along the similar lines described above one can identify more than one third order $O \Delta E$ possessing 2 independent integrals since the factorisation of $\tilde{F}$ and $\tilde{G}$ explained earlier is not unique. For example $\tilde{F}$ and $\tilde{G}$ can also be factored as

$$
\begin{aligned}
& \tilde{F}=2\left(\tilde{\beta}_{3} w_{1}+\tilde{\beta}_{3} w_{2}+\tilde{\beta}_{4}\right)\left[\tilde{\beta}_{3}\left(w_{1}+w_{2}\right)^{2}+\tilde{\gamma}_{7}\left(w_{1}+w_{2}\right)+\tilde{\gamma}_{9}\right] \\
& \tilde{G}=\left(\tilde{\beta}_{3} w_{1}+\tilde{\beta}_{3} w_{2}+\tilde{\beta}_{4}\right)^{2}
\end{aligned}
$$

provided

$$
\begin{aligned}
& \tilde{a}_{3}=\tilde{\beta}_{3}^{2}, \tilde{b}_{2}=2 \tilde{a}_{3}, \tilde{b}_{3}=\tilde{c}_{2}=2 \tilde{\beta}_{3} \tilde{\beta}_{4}, \tilde{c}_{3}=\tilde{\beta}_{4}^{2}, \\
& \epsilon_{3}=4 \tilde{a}_{3}, \epsilon_{4}=2 \tilde{\beta}_{3}\left(\tilde{\beta}_{4}+\tilde{\gamma}_{7}\right), \epsilon_{5}=\tilde{\beta}_{3} \tilde{\gamma}_{9}+2 \tilde{\beta}_{4}^{2}, \epsilon_{7}=2 \tilde{a}_{3}, \epsilon_{8}=2 \tilde{\beta}_{3} \tilde{\gamma}_{7}, \\
& \epsilon_{9}=2\left(\tilde{\beta}_{4} \tilde{\gamma}_{7}+\tilde{\beta}_{3} \tilde{\gamma}_{9}\right)-\epsilon_{5}, \epsilon_{10}=\tilde{\beta}_{4} \tilde{\gamma}_{9}, \epsilon_{12}=2 \beta_{3}^{2}, \epsilon_{13}=2 \tilde{\beta}_{3} \tilde{\gamma}_{7}, \\
& \epsilon_{14}=2\left(\tilde{\beta}_{4} \tilde{\gamma}_{7}+\tilde{\beta}_{3} \tilde{\gamma}_{9}\right)-\epsilon_{5}, \epsilon_{15}=\tilde{\beta}_{4} \tilde{\gamma}_{9}, \epsilon_{16}=\tilde{\beta}_{3}^{2}, \epsilon_{17}=2 \tilde{\beta}_{3}\left(\tilde{\gamma}_{7}-\tilde{\beta}_{4}\right), \\
& \epsilon_{18}=-2 \tilde{\beta}_{4} \tilde{\gamma}_{7}+\tilde{\beta}_{3} \tilde{\gamma}_{9}+\tilde{\gamma}_{7}^{2}+\tilde{\beta}_{4}^{2}, \epsilon_{19}=\tilde{\gamma}_{9}\left(\tilde{\gamma}_{7}-\tilde{\beta}_{4}\right), \\
& \tilde{a}_{1}=\tilde{a}_{2}=\epsilon_{1}=\epsilon_{2}=\epsilon_{6}=\epsilon_{11}=\epsilon_{20}=0 .
\end{aligned}
$$

Here again the conditions (2.5ab) and (2.10) are identically satisfied for the above parametric restrictions. In this case equation (2.20) becomes

$$
w_{n+3}=-w_{n}-\frac{\lambda_{1}\left(w_{n+1}+w_{n+2}\right)^{2}+\lambda_{2}\left(w_{n+1}+w_{n+2}\right)+\lambda_{3}}{\lambda_{1}\left(w_{n+1}+w_{n+2}\right)+\lambda_{4}},
$$

where

$$
\tilde{\beta}_{3}=\lambda_{1}, \tilde{\gamma}_{7}=\lambda_{2}, \tilde{\gamma}_{9}=\lambda_{3}, \tilde{\beta}_{4}=\lambda_{4}
$$


and admits second integral $I_{2}$ as

$$
\begin{aligned}
I_{2}= & {\left[\lambda_{1} w_{n} w_{n+2}+\lambda_{4} w_{n+2}+\lambda_{1} w_{n} w_{n+1}+\lambda_{4} w_{n}+\lambda_{1} w_{n+1}^{2}+\lambda_{1} w_{n+1} w_{n+2}\right.} \\
& \left.+\left(\lambda_{2}-\lambda_{4}\right) w_{n+1}\right]\left[\lambda_{1} w_{n} w_{n+2}+\lambda_{4} w_{n+2}+\lambda_{1} w_{n} w_{n+1}+\lambda_{4} w_{n}+\lambda_{1} w_{n+1}^{2}\right. \\
& \left.+\lambda_{1} w_{n+1} w_{n+2}+\left(\lambda_{2}-\lambda_{4}\right) w_{n+1}+\lambda_{3}\right] .
\end{aligned}
$$

It is easy to see that equation (2.14) possessing an integral $I_{1}$ takes exactly the same form as $(2.25)$ for the parametric restrictions

$$
c_{2}=b_{3}=\lambda_{1}, e_{2}=2 \lambda_{1}, e_{3}=\lambda_{2}, f_{3}=\lambda_{3}, c_{3}=\lambda_{4}, a_{1}=a_{2}=b_{2}=a_{3}=0
$$

and the integral $I_{1}$ becomes

$$
\begin{aligned}
I_{1} & =\left[\lambda_{1} w_{n}+\lambda_{1} w_{n+1}+\lambda_{4}\right] w_{n+2}^{2}+\left[\lambda_{1} w_{n}^{2}+2 \lambda_{1} w_{n} w_{n+1}+\lambda_{2} w_{n}+\lambda_{1} w_{n+1}^{2}+\lambda_{2} w_{n+1}\right. \\
& \left.+\lambda_{3}\right] w_{n+2}+\lambda_{1} w_{n+1} w_{n}^{2}+\lambda_{4} w_{n}^{2}+\lambda_{1} w_{n+1}^{2} w_{n}+\lambda_{2} w_{n+1} w_{n}+\lambda_{3} w_{n}+\lambda_{4} w_{n+1}^{2}+\lambda_{3} w_{n+1} .
\end{aligned}
$$

A detailed calculation shows that there exists no other third order difference equation possessing 2 independent polynomial integrals. We wish to mention that the identified third order difference equations (2.22) and (2.25) admitting 2 integrals are also measure preserving with measure 1 and hence they are integrable. Equations (2.22) and (2.25) were also obtained by Iatrou(2003a) using a different method. However, our analysis shows that there exists a third order difference equation involving atleast 10 parameters possessing one cyclic integral $I_{1}$.

\section{Construction of integrals for fourth order autonomous ordinary difference equation}

Consider an autonomous fourth order $O \Delta E$ having the form

$$
w_{4}=F\left(w_{0}, w_{1}, w_{2}, w_{3}\right) .
$$

Assume that equation (3.1) admits an integral $I\left(w_{0}, w_{1}, w_{2}, w_{3}\right)$ having the form

$$
I\left(w_{0}, w_{1}, w_{2}, w_{3}\right)=\sum_{j=1}^{3}\left[A_{1 j}\left(w_{1}, w_{2}\right) w_{3}^{2}+A_{2 j}\left(w_{1}, w_{2}\right) w_{3}+A_{3 j}\left(w_{1}, w_{2}\right)\right] w_{0}^{3-j} .
$$

Then the integrability condition $I(n+1)-I(n)=0$ leads to a quadratic equation in $w_{4}$

$$
\begin{aligned}
& F_{1}\left(w_{1}, w_{2}, w_{3}\right) w_{4}^{2}+F_{2}\left(w_{1}, w_{2}, w_{3}\right) w_{4} \\
& \quad-\left[F_{4}\left(w_{1}, w_{2}, w_{3}\right) w_{0}^{2}+F_{5}\left(w_{1}, w_{2}, w_{3}\right) w_{0}+\left(F_{6}\left(w_{1}, w_{2}, w_{3}\right)-F_{3}\left(w_{1}, w_{2}, w_{3}\right)\right)\right]=0,
\end{aligned}
$$

where

$$
\begin{aligned}
& F_{i}\left(w_{1}, w_{2}, w_{3}\right)=\sum_{j=1}^{3} A_{i j}\left(w_{2}, w_{3}\right) w_{1}^{3-j}, i=1,2,3, \\
& F_{k+3}\left(w_{1}, w_{2}, w_{3}\right)=\sum_{j=1}^{3} A_{j k}\left(w_{1}, w_{2}\right) w_{3}^{3-j}, k=1,2,3 .
\end{aligned}
$$


Hereafter we denote $F_{i}\left(w_{1}, w_{2}, w_{3}\right)$ as $F_{i}$ for the remaining section unless otherwise specified. Equation (3.3) can be factorised as

$$
\left(w_{4}+w_{0}+\frac{F_{5}+F_{2}}{2 F_{1}}\right)\left(w_{4}-w_{0}+\frac{F_{2}-F_{5}}{2 F_{1}}\right)=0
$$

provided

$$
\begin{aligned}
& A_{11}\left(w_{2}, w_{3}\right) w_{1}^{2}+A_{12}\left(w_{2}, w_{3}\right) w_{1}+A_{13}\left(w_{2}, w_{3}\right) \\
& \quad=A_{11}\left(w_{1}, w_{2}\right) w_{3}^{2}+A_{21}\left(w_{1}, w_{2}\right) w_{3}+A_{31}\left(w_{1}, w_{2}\right)
\end{aligned}
$$

and

$$
\left(F_{5}+F_{2}\right)\left(F_{5}-F_{2}\right)=4\left(F_{6}-F_{3}\right) F_{1} .
$$

Thus we obtain,

$$
\begin{aligned}
& w_{4}=-w_{0}-\frac{F_{2}+F_{5}}{2 F_{1}}, \\
& w_{4}=w_{0}-\frac{F_{2}-F_{5}}{2 F_{1}} .
\end{aligned}
$$

It is easy to check that equation (3.5a) is satisfied with

$$
\begin{aligned}
& A_{11}\left(w_{1}, w_{2}\right)=\left(a_{1} w_{1}^{2}+a_{2} w_{1}+a_{3}\right) w_{2}^{2}+\left(a_{2} w_{1}^{2}+a_{5} w_{1}+a_{6}\right) w_{2}+a_{3} w_{1}^{2}+a_{8} w_{1}+a_{9} \\
& A_{12}\left(w_{1}, w_{2}\right)=\left(a_{2} w_{1}^{2}+a_{5} w_{1}+a_{8}\right) w_{2}^{2}+A_{122}\left(w_{1}\right) w_{2}+A_{123}\left(w_{1}\right) \\
& A_{13}\left(w_{1}, w_{2}\right)=\left(a_{3} w_{1}^{2}+a_{6} w_{1}+a_{9}\right) w_{2}^{2}+A_{132}\left(w_{1}\right) w_{2}+A_{133}\left(w_{1}\right) \\
& A_{21}\left(w_{1}, w_{2}\right)=\left(a_{2} w_{2}^{2}+a_{5} w_{2}+a_{6}\right) w_{1}^{2}+A_{122}\left(w_{2}\right) w_{1}+A_{132}\left(w_{2}\right) \\
& A_{31}\left(w_{1}, w_{2}\right)=\left(a_{3} w_{2}^{2}+a_{8} w_{2}+a_{9}\right) w_{1}^{2}+A_{123}\left(w_{2}\right) w_{1}+A_{133}\left(w_{2}\right)
\end{aligned}
$$

where $a_{i}$ 's are constants while $A_{122}, A_{123}, A_{132}$ and $A_{133}$ are arbitrary functions. As pointed out for third order difference equations, equation $(3.5 \mathrm{~b})$ can be solved for two distinct possibilities:

(i) $\quad F_{5}=F_{2}$ and $F_{6}=F_{3}$,

(ii) $\quad F_{5} \neq F_{2}$ and $F_{6} \neq F_{3}$.

Considering the condition (3.9) we have

$$
\begin{aligned}
& A_{11}\left(w_{1}, w_{2}\right)=\left(a_{1} w_{1}^{2}+a_{2} w_{1}+a_{3}\right) w_{2}^{2}+\left(a_{2} w_{1}^{2}+a_{5} w_{1}+a_{6}\right) w_{2}+a_{3} w_{1}^{2}+a_{8} w_{1}+a_{9} \\
& A_{12}\left(w_{1}, w_{2}\right)=\left(a_{2} w_{1}^{2}+a_{5} w_{1}+a_{8}\right) w_{2}^{2}+\left(b_{4} w_{1}^{2}+b_{5} w_{1}+b_{6}\right) w_{2}+b_{7} w_{1}^{2}+b_{8} w_{1}+b_{9} \\
& A_{13}\left(w_{1}, w_{2}\right)=\left(a_{3} w_{1}^{2}+a_{6} w_{1}+a_{9}\right) w_{2}^{2}+\left(b_{7} w_{1}^{2}+c_{5} w_{1}+c_{6}\right) w_{2}+c_{7} w_{1}^{2}+c_{8} w_{1}+c_{9} \\
& A_{21}\left(w_{1}, w_{2}\right)=\left(a_{2} w_{1}^{2}+b_{4} w_{1}+b_{7}\right) w_{2}^{2}+\left(a_{5} w_{1}^{2}+b_{5} w_{1}+c_{5}\right) w_{2}+a_{6} w_{1}^{2}+b_{6} w_{1}+c_{6}
\end{aligned}
$$




$$
\begin{aligned}
& A_{22}\left(w_{1}, w_{2}\right)=\left(a_{5} w_{1}^{2}+b_{5} w_{1}+b_{8}\right) w_{2}^{2}+\left(b_{5} w_{1}^{2}+e_{5} w_{1}+e_{6}\right) w_{2}+c_{5} w_{1}^{2}+e_{6} w_{1}+e_{9} \\
& A_{23}\left(w_{1}, w_{2}\right)=\left(a_{8} w_{1}^{2}+b_{6} w_{1}+b_{9}\right) w_{2}^{2}+\left(b_{8} w_{1}^{2}+e_{6} w_{1}+e_{9}\right) w_{2}+c_{8} w_{1}^{2}+f_{8} w_{1}+f_{9} \\
& (3.11) \\
& A_{31}\left(w_{1}, w_{2}\right)=\left(a_{3} w_{1}^{2}+b_{7} w_{1}+c_{7}\right) w_{2}^{2}+\left(a_{8} w_{1}^{2}+b_{8} w_{1}+c_{8}\right) w_{2}+a_{9} w_{1}^{2}+b_{9} w_{1}+c_{9} \\
& \\
& A_{32}\left(w_{1}, w_{2}\right)=\left(a_{6} w_{1}^{2}+c_{5} w_{1}+c_{8}\right) w_{2}^{2}+\left(b_{6} w_{1}^{2}+e_{6} w_{1}+f_{8}\right) w_{2}+c_{6} w_{1}^{2}+e_{9} w_{1}+f_{9} \\
& A_{33}\left(w_{1}, w_{2}\right)=\left(a_{9} w_{1}^{2}+c_{6} w_{1}+c_{9}\right) w_{2}^{2}+\left(b_{9} w_{1}^{2}+e_{9} w_{1}+f_{9}\right) w_{2}+c_{9} w_{1}^{2}+f_{9} w_{1}+j_{9}
\end{aligned}
$$

where $a_{i}$ 's, $b_{i}$ 's, $c_{i}$ 's, $e_{i}$ 's, $f_{i}$ 's and $j_{9}$ are arbitrary constants. Thus we obtain a fourth order difference equation

$$
w_{4}=-w_{0}-\frac{F_{2}+F_{5}}{2 F_{1}}=-w_{0}-\frac{F}{G}
$$

where

$$
\begin{aligned}
F & =\left[\left(a_{2} w_{1}^{2}+a_{5} w_{1}+a_{8}\right) w_{2}^{2}+\left(b_{4} w_{1}^{2}+b_{5} w_{1}+b_{6}\right) w_{2}+b_{7} w_{1}^{2}+b_{8} w_{1}+b_{9}\right] w_{3}^{2} \\
& +\left[\left(a_{5} w_{1}^{2}+b_{5} w_{1}+b_{8}\right) w_{2}^{2}+\left(b_{5} w_{1}^{2}+e_{5} w_{1}+e_{6}\right) w_{2}+c_{5} w_{1}^{2}+e_{6} w_{1}+e_{9}\right] w_{3} \\
& +\left[\left(a_{6} w_{1}^{2}+c_{5} w_{1}+c_{8}\right) w_{2}^{2}+\left(b_{6} w_{1}^{2}+e_{6} w_{1}+f_{8}\right) w_{2}+c_{6} w_{1}^{2}+e_{9} w_{1}+f_{9}\right] \\
G & =\left[\left(a_{1} w_{1}^{2}+a_{2} w_{1}+a_{3}\right) w_{2}^{2}+\left(a_{2} w_{1}^{2}+a_{5} w_{1}+a_{6}\right) w_{2}+a_{3} w_{1}^{2}+a_{8} w_{1}+a_{9}\right] w_{3}^{2} \\
& +\left[\left(a_{5} w_{1}^{2}+b_{5} w_{1}+c_{5}\right) w_{2}+c_{6}+\left(b_{4} w_{1}+b_{7}+a_{2} w_{1}^{2}\right) w_{2}^{2}+b_{6} w_{1}+a_{6} w_{1}^{2}\right] w_{3} \\
& +\left(a_{3} w_{1}^{2}+b_{7} w_{1}+c_{7}\right) w_{2}^{2}+c_{9}+a_{9} w_{1}^{2}+\left(a_{8} w_{1}^{2}+b_{8} w_{1}+c_{8}\right) w_{2}+b_{9} w_{1}
\end{aligned}
$$

with an integral $I_{1}$ given by

$$
\begin{aligned}
I_{1} & =\left[\left[\left(a_{1} w_{1}^{2}+a_{2} w_{1}+a_{3}\right) w_{2}^{2}+\left(a_{2} w_{1}^{2}+a_{5} w_{1}+a_{6}\right) w_{2}+a_{3} w_{1}^{2}+a_{8} w_{1}+a_{9}\right] w_{0}^{2}\right. \\
& +\left[\left(a_{2} w_{1}^{2}+a_{5} w_{1}+a_{8}\right) w_{2}^{2}+\left(b_{4} w_{1}^{2}+b_{5} w_{1}+b_{6}\right) w_{2}+b_{7} w_{1}^{2}+b_{8} w_{1}+b_{9}\right] w_{0} \\
& \left.+\left(a_{3} w_{1}^{2}+a_{6} w_{1}+a_{9}\right) w_{2}^{2}+\left(b_{7} w_{1}^{2}+c_{5} w_{1}+c_{6}\right) w_{2}+c_{7} w_{1}^{2}+c_{8} w_{1}+c_{9}\right] w_{3}^{2} \\
& +\left[\left[\left(a_{2} w_{1}^{2}+b_{4} w_{1}+b_{7}\right) w_{2}^{2}+\left(a_{5} w_{1}^{2}+b_{5} w_{1}+c_{5}\right) w_{2}+a_{6} w_{1}^{2}+b_{6} w_{1}+c_{6}\right] w_{0}^{2}\right. \\
& +\left[\left(a_{5} w_{1}^{2}+b_{5} w_{1}+b_{8}\right) w_{2}^{2}+\left(b_{5} w_{1}^{2}+e_{5} w_{1}+e_{6}\right) w_{2}+c_{5} w_{1}^{2}+e_{6} w_{1}+e_{9}\right] w_{0} \\
& \left.+\left(a_{8} w_{1}^{2}+b_{6} w_{1}+b_{9}\right) w_{2}^{2}+\left(b_{8} w_{1}^{2}+e_{6} w_{1}+e_{9}\right) w_{2}+c_{8} w_{1}^{2}+f_{8} w_{1}+f_{9}\right] w_{3} \\
& +\left[\left(a_{3} w_{1}^{2}+b_{7} w_{1}+c_{7}\right) w_{2}^{2}+\left(a_{8} w_{1}^{2}+b_{8} w_{1}+c_{8}\right) w_{2}+a_{9} w_{1}^{2}+b_{9} w_{1}+c_{9}\right] w_{0}^{2} \\
& +\left[\left(a_{6} w_{1}^{2}+c_{5} w_{1}+c_{8}\right) w_{2}^{2}+\left(b_{6} w_{1}^{2}+e_{6} w_{1}+f_{8}\right) w_{2}+c_{6} w_{1}^{2}+e_{9} w_{1}+f_{9}\right] w_{0} \\
& +\left(a_{9} w_{1}^{2}+c_{6} w_{1}+c_{9}\right) w_{2}^{2}+\left(b_{9} w_{1}^{2}+e_{9} w_{1}+f_{9}\right) w_{2}+c_{9} w_{1}^{2}+f_{9} w_{1}
\end{aligned}
$$

Proceeding further along the lines described for third order $O \Delta E$ we find that there exist six fourth order $O \Delta E$ possessing 2 independent integrals. They are

(i) $w_{n+4}=-w_{n}-\frac{\lambda_{1}\left(w_{n+2}^{2}+w_{n+2}\left(w_{n+1}+w_{n+3}\right)\right)+\lambda_{3}\left(w_{n+1}+w_{n+3}\right)+\lambda_{2} w_{n+2}+\lambda_{4}}{\lambda_{1} w_{n+2}+\lambda_{2}}$, 


$$
\begin{aligned}
I_{1} & =\left(\lambda_{1} w_{n+1}+\lambda_{3}\right) w_{n+3}^{2}+\left(\left(2 \lambda_{1} w_{n+1}+2 \lambda_{3}\right) w_{n+2}+\lambda_{1} w_{n+1}^{2}+\lambda_{2} w_{n+1}+\lambda_{4}\right) w_{n+3} \\
& +\left(\lambda_{1} w_{n+1}+2 \lambda_{3}\right) w_{n+2}^{2}+\left(\lambda_{1} w_{n+1}^{2}+\left(\lambda_{2}+2 \lambda_{3}\right) w_{n+1}+2 \lambda_{4}\right) w_{n+2}+2 \lambda_{3} w_{n+1}^{2} \\
& +2 \lambda_{4} w_{n+1}+\left(\lambda_{1} w_{n+2}+\lambda_{3}\right) w_{n}^{2}+\left(\left(2 \lambda_{1} w_{n+2}+2 \lambda_{3}\right) w_{n+1}+\lambda_{1} w_{n+2}^{2}+\lambda_{2} w_{n+2}+\lambda_{4}\right) w_{n} .
\end{aligned}
$$

$$
w_{n+4}=-w_{n}-\frac{\lambda_{1}\left(w_{n+1}+w_{n+2}+w_{n+3}\right)^{2}+\lambda_{2}\left(w_{n+1}+w_{n+2}+w_{n+3}\right)+\lambda_{3}}{\lambda_{1}\left(w_{n+1}+w_{n+2}+w_{n+3}\right)+\lambda_{4}},
$$

$$
\begin{aligned}
I_{1} & =\left(\lambda_{1} w_{n}+\lambda_{1} w_{n+2}+\lambda_{1} w_{n+1}+\lambda_{4}\right) w_{n+3}^{2}+\left(\lambda_{1} w_{n}^{2}+\left(2 \lambda_{1} w_{n+2}+2 \lambda_{1} w_{n+1}+\lambda_{2}\right) w_{n}\right. \\
& \left.+\lambda_{1} w_{n+2}^{2}+\left(2 \lambda_{1} w_{n+1}+\lambda_{2}\right) w_{n+2}+\lambda_{1} w_{n+1}^{2}+\lambda_{2} w_{n+1}+\lambda_{3}\right) w_{n+3}+\left(\lambda_{1} w_{n+1}\right. \\
& \left.+\lambda_{4}\right) w_{n+2}^{2}+\left(\lambda_{1} w_{n+1}^{2}+\lambda_{2} w_{n+1}+\lambda_{3}\right) w_{n+2}+\lambda_{4} w_{n+1}^{2}+\lambda_{3} w_{n+1}+\left(\lambda_{1} w_{n+2}\right. \\
& \left.+\lambda_{1} w_{n+1}+\lambda_{4}\right) w_{n}^{2}+\left(\lambda_{1} w_{n+1}^{2}+\left(2 \lambda_{1} w_{n+2}+\lambda_{2}\right) w_{n+1}+\lambda_{1} w_{n+2}^{2}+\lambda_{2} w_{n+2}+\lambda_{3}\right) w_{n} .
\end{aligned}
$$

$($ iii)

$$
w_{n+4}=-w_{n}-\frac{\lambda_{1}\left(w_{n+1} w_{n+2}+w_{n+2} w_{n+3}\right)+\lambda_{2}\left(w_{n+1}+w_{n+3}\right)+\lambda_{4} w_{n+2}+\lambda_{3}}{\lambda_{1}\left(w_{n+1}+w_{n+3}\right)+\lambda_{4}},
$$

$$
\begin{aligned}
I_{1}= & \left(\lambda_{1} w_{n}+\lambda_{2}-\lambda_{4}\right)\left(\lambda_{1} w_{n+2}+\lambda_{2}-\lambda_{4}\right)\left(\lambda_{1} w_{n+1}+\lambda_{2}-\lambda_{4}\right)\left[\left(\lambda_{1} w_{n}+\lambda_{1} w_{n+2}+\lambda_{4}\right) w_{n+3}\right. \\
& \left.+\lambda_{1} w_{n} w_{n+1}+\lambda_{1} w_{n+1} w_{n+2}+\lambda_{4} w_{n+1}+\lambda_{2} w_{n+2}+\lambda_{2} w_{n}+\lambda_{3}\right] w_{n+3}+\left(\lambda_{2}-\lambda_{4}\right) \\
& \times\left(\lambda_{1} w_{n+2}+\lambda_{2}-\lambda_{4}\right)\left(\lambda_{1} w_{n+1}+\lambda_{2}-\lambda_{4}\right)\left(\lambda_{1} w_{n+1} w_{n+2}+\lambda_{1} w_{n} w_{n+1}+\lambda_{2} w_{n+1}\right. \\
& \left.+\lambda_{4} w_{n+2}+\lambda_{4} w_{n}+\lambda_{3}\right) w_{n}+\left(\lambda_{2}-\lambda_{4}\right)^{2}\left(\lambda_{2}^{2} w_{n+1} w_{n+2}+\lambda_{2} \lambda_{4} w_{n+2}^{2}+\lambda_{2} \lambda_{3} w_{n+2}\right. \\
& -\lambda_{2} \lambda_{4} w_{n+1} w_{n+2}+\lambda_{1} \lambda_{2} w_{n+1} w_{n+2}^{2}+\lambda_{1} \lambda_{2} w_{n+1}^{2} w_{n+2}+\lambda_{2} \lambda_{4} w_{n+1}^{2}+\lambda_{2} \lambda_{3} w_{n+1} \\
& \left.-\lambda_{4} \lambda_{3} w_{n+2}+\lambda_{1}^{2} w_{n+1}^{2} w_{n+2}^{2}-\lambda_{4}^{2} w_{n+2}^{2}+\lambda_{1} \lambda_{3} w_{n+1} w_{n+2}-\lambda_{4}^{2} w_{n+1}^{2}-\lambda_{3} \lambda_{4} w_{n+1}\right) .
\end{aligned}
$$

(iv) $w_{n+4}=-w_{n}-\frac{\left(\lambda_{1} w_{n+2}+2 \lambda_{3}\right)\left(w_{n+1}+w_{n+3}\right)+\lambda_{1}\left(w_{n+2}^{2}+w_{n+1} w_{n+3}\right)+\lambda_{2} w_{n+2}+\lambda_{4}}{\lambda_{1} w_{n+2}+\lambda_{3}}$,

$$
\begin{aligned}
I_{1} & =\left(\lambda_{1} w_{n+1}+\lambda_{3}\right) w_{n+3}^{2}+\left(\left(\lambda_{1} w_{n+2}+\lambda_{1} w_{n+1}+2 \lambda_{3}\right) w_{n}+\left(\lambda_{1} w_{n+1}+2 \lambda_{3}\right) w_{n+2}\right. \\
& \left.+\lambda_{1} w_{n+1}^{2}+\lambda_{2} w_{n+1}+\lambda_{4}\right) w_{n+3}+\lambda_{3} w_{n+2}^{2}+\left(2 \lambda_{3} w_{n+1}+\lambda_{4}\right) w_{n+2}+\lambda_{3} w_{n+1}^{2} \\
& +\lambda_{4} w_{n+1}+\left(\lambda_{1} w_{n+2}+\lambda_{3}\right) w_{n}^{2}+\left(\left(\lambda_{1} w_{n+2}+2 \lambda_{3}\right) w_{n+1}+\lambda_{1} w_{n+2}^{2}+\lambda_{2} w_{n+2}+\lambda_{4}\right) w_{n} .
\end{aligned}
$$

(v) $w_{n+4}=-w_{n}-\frac{\lambda_{1}\left(w_{n+1}^{2}+2 w_{n+2}^{2}+w_{n+3}^{2}+4\left(w_{n+1} w_{n+2}+w_{n+2} w_{n+3}+w_{n+3} w_{n+1}\right)\right)}{\lambda_{1} w_{n+1}+\lambda_{1} w_{n+3}+2 \lambda_{1} w_{n+2}+\lambda_{3}}$

$$
-\frac{2\left(\lambda_{2}-\lambda_{3}\right) w_{n+2}+\lambda_{2}\left(w_{n+1}+w_{n+3}\right)+\lambda_{4}}{\lambda_{1} w_{n+1}+\lambda_{1} w_{n+3}+2 \lambda_{1} w_{n+2}+\lambda_{3}}
$$




$$
\begin{aligned}
I_{1} & =\left(\lambda_{1} w_{n}+\lambda_{1} w_{n+2}+2 \lambda_{1} w_{n+1}+\lambda_{3}\right) w_{n+3}^{2}+\left(\lambda_{1} w_{n}^{2}+\left(4 \lambda_{1} w_{n+2}+4 \lambda_{1} w_{n+1}+\lambda_{2}\right) w_{n}\right. \\
& \left.+\lambda_{1} w_{n+2}^{2}+\left(4 \lambda_{1} w_{n+1}+\lambda_{2}\right) w_{n+2}+2 \lambda_{1} w_{n+1}^{2}+\left(2 \lambda_{2}-2 \lambda_{3}\right) w_{n+1}+\lambda_{4}\right) w_{n+3}+\left(\lambda_{1} w_{n+1}\right. \\
& \left.+\lambda_{3}\right) w_{n+2}^{2}+\left(\lambda_{1} w_{n+1}^{2}+\lambda_{2} w_{n+1}+\lambda_{4}\right) w_{n+2}+\lambda_{3} w_{n+1}^{2}+\lambda_{4} w_{n+1}+\left(\lambda_{1} w_{n+1}+2 \lambda_{1} w_{n+2}\right. \\
& \left.+\lambda_{3}\right) w_{n}^{2}+\left(\lambda_{1} w_{n+1}^{2}+\left(4 \lambda_{1} w_{n+2}+\lambda_{2}\right) w_{n+1}+2 \lambda_{1} w_{n+2}^{2}+\left(2 \lambda_{2}-2 \lambda_{3}\right) w_{n+2}+\lambda_{4}\right) w_{n} .
\end{aligned}
$$

$$
\begin{aligned}
& w_{n+4}=-w_{n}-\frac{\lambda_{1} w_{n+1} w_{n+3}+2 \lambda_{4} w_{n+2}+\left(\lambda_{4}+\lambda_{5}\right)\left(w_{n+1}+w_{n+3}\right)+\lambda_{3}}{\lambda_{1} w_{n+2}+\lambda_{4}-\lambda_{5}}, \\
& I_{1}=\left(\lambda_{1} w_{n+1}+\lambda_{4}-\lambda_{5}\right) w_{n+3}^{2}+\left(\left(\lambda_{1} w_{n+2}+\lambda_{1} w_{n+1}+2 \lambda_{4}\right) w_{n}+\left(2 \lambda_{5}-\lambda_{1} w_{n+1}\right) w_{n+2}\right. \\
& \left.+2 \lambda_{4} w_{n+1}+\lambda_{3}\right) w_{n+3}-2 \lambda_{5}\left(w_{n+1}^{2}+w_{n+2}^{2}\right)+2\left(\lambda_{5}-\lambda_{4}\right) w_{n+1} w_{n+2}+\left(\lambda_{1} w_{n+2}\right. \\
& \left.+\lambda_{4}-\lambda_{5}\right) w_{n}^{2}+\left(\left(2 \lambda_{5}-\lambda_{1} w_{n+2}\right) w_{n+1}+2 \lambda_{4} w_{n+2}+\lambda_{3}\right) w_{n} .
\end{aligned}
$$

Since the calculations are tedious and cumbersome the explicit forms of expressions $A_{i j}\left(w_{1}, w_{2}\right)$ associated with second integral $I_{2}$ for each of the above identified six cases are given in Appendix A.

Finally we would like to mention that with the limited software facilities available we have identified the above fourth order $O \Delta E$ possessing 2 independent polynomial integrals and hence we do not claim that the identified list of difference equations is an exhaustive one.

\section{Summary}

In this article a method is described to construct polynomial integrals for third order and fourth order $O \Delta E$. We have identified two distinct third order equations (2.22) and (2.25), each of them admits 2 independent integrals. It is appropriate to mention here that one of the integrals in both the cases is cyclic invariant. Also the identified equations $(2.22)$ and (2.25) are measure preserving with measure 1 and hence they are integrable. Similar observation has also been noted by Iatrou (2003a). For fourth order $O \Delta E$ we have identified six distinct equations (3.14), (3.15), (3.16), (3.17), (3.18) and (3.19) admitting 2 independent integrals. Here again one of the two integrals is cyclic invariant. Also they are reversible as well as measure preserving with measure 1 . We would like to mention that the fourth order equations given in equations (3.14), (3.15) and (3.16) are also symplectic and hence they are integrable in the Liouville sense. This was also observed by Iatrou(2003b). We wish to recall that Byrnes et al (1999) has proved that if an $N$-th order autonomous difference equation is measure preserving and has at least $N-2$ integrals then it has a degenerate Poisson structure, so defines a symplectic map on each $2 D$ level set of these integrals. Difference equations given by (3.17), (3.18), (3.19) possessing 2 independent integrals belong to this category and they are expected to be integrable. The integrability of the above difference equations can be established(hopefully) by other means, for example through a criterion developed for discrete equations (Halburd,2005). The effectiveness of the method is also discussed for $N$-th order $O \Delta E$ in the Appendix.

Acknowledgments. The work of R.S. forms part of the research project funded by CSIR, New Delhi. The work of C.U. is supported by UGC, New Delhi. 


\section{Appendix A}

From the analysis we observe that $A_{21}\left(w_{n+1}, w_{n+2}\right)=A_{12}\left(w_{n+2}, w_{n+1}\right), A_{32}\left(w_{n+1}, w_{n+2}\right)=$ $A_{23}\left(w_{n+2}, w_{n+1}\right), A_{31}\left(w_{n+1}, w_{n+2}\right)=A_{13}\left(w_{n+2}, w_{n+1}\right)$ in all the six fourth order difference equations and therefore we presented only the forms of $A_{11}\left(w_{n+1}, w_{n+2}\right), A_{12}\left(w_{n+1}, w_{n+2}\right)$, $A_{13}\left(w_{n+1}, w_{n+2}\right), A_{22}\left(w_{n+1}, w_{n+2}\right), A_{23}\left(w_{n+1}, w_{n+2}\right), A_{33}\left(w_{n+1}, w_{n+2}\right)$.

Explicit forms for $A_{i j}\left(w_{n+1}, w_{n+2}\right)$ associated with fourth order difference equation (3.14):

$$
\begin{aligned}
A_{11} & =0, \\
A_{12}= & \lambda_{1}\left(\lambda_{1} w_{n+1}+\lambda_{3}\right)\left(\lambda_{1} w_{n+2}+\lambda_{3}\right), \\
A_{13}= & \left(\lambda_{1} w_{n+1}+\lambda_{3}\right)\left(\left(\lambda_{1} \lambda_{3} w_{n+2}+\lambda_{1} \lambda_{4}\right)+2 \lambda_{3}^{2}-\lambda_{2} \lambda_{3}\right), \\
A_{22}= & \left(2 \lambda_{3}^{2}-\lambda_{2} \lambda_{3}+\lambda_{1} \lambda_{3} w_{n+2}+\lambda_{1} \lambda_{4}\right)\left(\lambda_{1} w_{n+1}+\lambda_{3}\right)+\lambda_{1}\left(\lambda_{1} w_{n+2}+\lambda_{3}\right) \\
& \times\left(\lambda_{4}+\lambda_{1} w_{n+1}^{2}+\lambda_{1} w_{n+1} w_{n+2}+\lambda_{2} w_{n+1}+\lambda_{3} w_{n+2}\right), \\
A_{23}= & \left(\lambda_{1} \lambda_{3} w_{n+2}+\lambda_{1} \lambda_{4}+2 \lambda_{3}^{2}-\lambda_{3} \lambda_{2}\right)\left(\lambda_{4}+\lambda_{1} w_{n+1}^{2}+\lambda_{1} w_{n+1} w_{n+2}+\lambda_{2} w_{n+1}+\lambda_{3} w_{n+2}\right), \\
A_{33}= & \left(2 \lambda_{3}^{2}-\lambda_{3} \lambda_{2}+\lambda_{1} \lambda_{4}\right)\left(\lambda_{3} w_{n+2}^{2}+\lambda_{4} w_{n+2}+\lambda_{4} w_{n+1}\right)+\lambda_{1} \lambda_{3}\left(\lambda_{3} w_{n+2}+\lambda_{3} w_{n+1}\right. \\
& \left.+\lambda_{4}\right) w_{n+1} w_{n+2}+\lambda_{3}\left(2 \lambda_{3}^{2}-\lambda_{3} \lambda_{2}+\lambda_{1} \lambda_{4}\right)\left(w_{n+2}+w_{n+1}\right) w_{n+1} .
\end{aligned}
$$

Explicit forms for $A_{i j}\left(w_{n+1}, w_{n+2}\right)$ associated with fourth order difference equation (3.15):

$$
\begin{aligned}
A_{11}= & \lambda_{1}^{2}\left(\lambda_{1} w_{n+2}+\lambda_{1} w_{n+1}-2 \lambda_{4}+\lambda_{2}\right), \\
A_{12}= & \lambda_{1}\left(\lambda_{1} w_{n+1}+\lambda_{1} w_{n+2}+\lambda_{4}\right)\left(\lambda_{1} w_{n+2}+\lambda_{1} w_{n+1}-2 \lambda_{4}+\lambda_{2}\right) \\
& +\lambda_{1}^{2}\left(\left(2 \lambda_{2}-4 \lambda_{4}\right) w_{n+1}+\lambda_{1} w_{n+1} w_{n+2}+\lambda_{1} w_{n+1}^{2}+\lambda_{2} w_{n+2}-2 \lambda_{4} w_{n+2}\right), \\
A_{13}= & \lambda_{1}\left(\lambda_{1} w_{n+1}+\lambda_{1} w_{n+2}+\lambda_{4}\right)\left(2 \lambda_{2} w_{n+1}-4 \lambda_{4} w_{n+1}+\lambda_{1} w_{n+1} w_{n+2}+\lambda_{1} w_{n+1}^{2}\right. \\
& \left.+\lambda_{2} w_{n+2}-2 \lambda_{4} w_{n+2}\right), \\
& =2 \lambda_{1}^{3} w_{n+2}^{3}+\lambda_{1}\left(\lambda_{1}\left(\lambda_{3}-16 \lambda_{4} w_{n+1}+7 \lambda_{1} w_{n+1}^{2}+11 \lambda_{2} w_{n+1}\right)+2 \lambda_{2}\left(\lambda_{2}-2 \lambda_{4}\right)\right) w_{n+2} \\
A_{22} & +\lambda_{1}^{2}\left(5 \lambda_{2}-7 \lambda_{4}+7 \lambda_{1} w_{n+1}\right) w_{n+2}^{2}+2 \lambda_{1}^{3} w_{n+1}^{3}+\lambda_{1}^{2}\left(5 \lambda_{2}-7 \lambda_{4}\right) w_{n+1}^{2}+\lambda_{1}\left(\lambda_{1} \lambda_{3}\right. \\
& \left.+2 \lambda_{2}^{2}-4 \lambda_{2} \lambda_{4}\right) w_{n+1}+\lambda_{1} \lambda_{2} \lambda_{3}+\lambda_{4}\left(4 \lambda_{2} \lambda_{4}-\lambda_{2}^{2}-4 \lambda_{4}^{2}-2 \lambda_{1} \lambda_{3}\right), \\
& =2 \lambda_{1}^{2}\left(\lambda_{1} w_{n+1}+\lambda_{2}-2 \lambda_{4}\right) w_{n+2}^{3}+\left(\lambda_{1}^{2}\left(5 \lambda_{1} w_{n+1}+8 \lambda_{2}-13 \lambda_{4}\right) w_{n+1}+\lambda_{1}\left(2 \lambda_{4}^{2}\right.\right. \\
A_{23} & \left.\left.+2 \lambda_{2}^{2}-5 \lambda_{2} \lambda_{4}\right)\right) w_{n+2}^{2}+\left(\lambda _ { 1 } \left(\lambda_{1}\left(4 \lambda_{1} w_{n+1}+9 \lambda_{2}-13 \lambda_{4}\right) w_{n+1}+4 \lambda_{2}^{2}-8 \lambda_{2} \lambda_{4}\right.\right. \\
& \left.\left.+\lambda_{1} \lambda_{3}\right) w_{n+1}+\lambda_{4}\left(4 \lambda_{4}^{2}-4 \lambda_{2} \lambda_{4}-2 \lambda_{1} \lambda_{3}+\lambda_{2}^{2}\right)+\lambda_{1} \lambda_{2} \lambda_{3}\right) w_{n+2}+\lambda_{1}^{3} w_{n+1}^{4} \\
& +\lambda_{1}^{2}\left(3 \lambda_{2}-4 \lambda_{4}\right) w_{n+1}^{3}+\lambda_{1}\left(\lambda_{1} \lambda_{3}+2 \lambda_{2}^{2}-4 \lambda_{2} \lambda_{4}\right) w_{n+1}^{2}+2 \lambda_{1} \lambda_{3}\left(\lambda_{2}-2 \lambda_{4}\right) w_{n+1},
\end{aligned}
$$




$$
\begin{aligned}
A_{33} & =\lambda_{1}^{2}\left(\lambda_{1} w_{n+1}+\lambda_{2}-2 \lambda_{4}\right) w_{n+2}^{4}+\left(3 \lambda_{1}^{3} w_{n+1}^{2}+\lambda_{1}^{2}\left(5 \lambda_{2}-8 \lambda_{4}\right) w_{n+1}+\lambda_{1}\left(2 \lambda_{2}^{2}+4 \lambda_{4}^{2}\right.\right. \\
& \left.\left.-6 \lambda_{2} \lambda_{4}\right)\right) w_{n+2}^{3}+\left(3 \lambda_{1}^{3} w_{n+1}^{3}+4 \lambda_{1}^{2}\left(2 \lambda_{2}-3 \lambda_{4}\right) w_{n+1}^{2}+\lambda_{1}\left(6 \lambda_{4}^{2}-13 \lambda_{2} \lambda_{4}+5 \lambda_{2}^{2}\right.\right. \\
& \left.\left.+\lambda_{1} \lambda_{3}\right) w_{n+1}+\lambda_{4}\left(4 \lambda_{2} \lambda_{4}-4 \lambda_{2}^{2}-2 \lambda_{1} \lambda_{3}\right)+\lambda_{2}\left(\lambda_{2}^{2}+\lambda_{1} \lambda_{3}\right)\right) w_{n+2}^{2}+\left(\lambda_{1}^{3} w_{n+1}^{4}\right. \\
& +\lambda_{1}^{2}\left(5 \lambda_{2}-8 \lambda_{4}\right) w_{n+1}^{3}+\lambda_{1}\left(6 \lambda_{4}^{2}-13 \lambda_{2} \lambda_{4}+5 \lambda_{2}^{2}+\lambda_{1} \lambda_{3}\right) w_{n+1}^{2}+\left(4 \lambda_{4}^{3}-6 \lambda_{1} \lambda_{3} \lambda_{4}\right. \\
& \left.\left.-3 \lambda_{2}^{2} \lambda_{4}+3 \lambda_{1} \lambda_{2} \lambda_{3}+\lambda_{2}^{3}\right) w_{n+1}+\lambda_{3}\left(4 \lambda_{4}^{2}-4 \lambda_{2} \lambda_{4}+\lambda_{2}^{2}\right)\right) w_{n+2} \\
& +w_{n+1}\left(\lambda_{2}-2 \lambda_{4}\right)\left(\lambda_{1} w_{n+1}+\lambda_{2}-2 \lambda_{4}\right)\left(\lambda_{1} w_{n+1}^{2}+\lambda_{2} w_{n+1}+\lambda_{3}\right) .
\end{aligned}
$$

Explicit forms for $A_{i j}\left(w_{n+1}, w_{n+2}\right)$ associated with fourth order difference equation (3.16):

$$
\begin{aligned}
A_{11}= & \lambda_{1}^{3}\left(\lambda_{1} w_{n+1} w_{n+2}+\left(\lambda_{2}-\lambda_{4}\right)\left(w_{n+1}+w_{n+2}\right)\right), \\
A_{12}= & \lambda_{1}^{2}\left(\lambda_{1} w_{n+2}+\lambda_{4}\right)\left(\lambda_{1} w_{n+1} w_{n+2}+\left(\lambda_{2}-\lambda_{4}\right)\left(w_{n+2}+w_{n+1}\right)\right) \\
& +\lambda_{1}\left(\lambda_{2}-\lambda_{4}\right)\left(\lambda_{1}^{2} w_{n+1} w_{n+2}+\left(\lambda_{2}-\lambda_{4}\right)\left(\lambda_{1} w_{n+1}-\lambda_{2}+\lambda_{4}\right)\right), \\
A_{13}= & \left(\lambda_{1} w_{n+2}+\lambda_{4}\right)\left(\lambda_{2}-\lambda_{4}\right)\left(\lambda_{1}^{2} w_{n+1} w_{n+2}+\left(\lambda_{2}-\lambda_{4}\right)\left(\lambda_{1} w_{n+1}+\lambda_{4}-\lambda_{2}\right)\right), \\
A_{22}= & \lambda_{1}^{2}\left(\lambda_{1} w_{n+1}+\lambda_{2}\right)\left(\lambda_{2}-\lambda_{4}+\lambda_{1} w_{n+1}\right) w_{n+2}^{2}+\lambda_{1}^{2}\left(\lambda_{1}\left(2 \lambda_{2}-\lambda_{4}\right) w_{n+1}^{2}\right. \\
& \left.+\left(\lambda_{1} \lambda_{3}+2 \lambda_{2}^{2}-\lambda_{2} \lambda_{4}-\lambda_{4}^{2}\right) w_{n+1}+\lambda_{3}\left(\lambda_{2}-\lambda_{4}\right)\right) w_{n+2} \\
& +\left(\lambda_{2}-\lambda_{4}\right)\left(\lambda_{1}^{2}\left(\lambda_{2} w_{n+1}+\lambda_{3}\right) w_{n+1}+\left(\lambda_{2}-\lambda_{4}\right)\left(\lambda_{4}^{2}-\lambda_{2}^{2}\right)\right), \\
& \\
A_{23}= & \lambda_{1}\left(\lambda_{2}-\lambda_{4}\right)\left(\lambda_{1}^{2} w_{n+1}^{2}+\lambda_{1}\left(2 \lambda_{2}-\lambda_{4}\right) w_{n+1}+\left(\lambda_{2}-\lambda_{4}\right)^{2}\right) w_{n+2}^{2} \\
& +\left(\lambda_{2}-\lambda_{4}\right)\left(\lambda_{1}^{2}\left(\lambda_{2} w_{n+1}+\lambda_{3}\right) w_{n+1}-\left(\lambda_{2}-\lambda_{4}\right)\left(\left(\lambda_{2}-\lambda_{4}\right)^{2}-2 \lambda_{1} \lambda_{4} w_{n+1}\right)\right) w_{n+2} \\
& +\left(\lambda_{2}-\lambda_{4}\right)^{2}\left(\lambda_{4} w_{n+1}+\lambda_{3}\right)\left(\lambda_{1} w_{n+1}-\lambda_{2}+\lambda_{4}\right), \\
& \\
A_{33}= & \left(\lambda_{2}-\lambda_{4}\right)^{2}\left(\left(\lambda_{1}^{2} w_{n+1}^{2}+\lambda_{1} \lambda_{2} w_{n+1}+\left(\lambda_{2}-\lambda_{4}\right)^{2}\right) w_{n+2}^{2}+\left(\lambda_{1} \lambda_{2} w_{n+1}^{2}\right.\right. \\
- & \left.\left.\left(\lambda_{2}^{2}-3 \lambda_{2} \lambda_{4}+2 \lambda_{4}^{2}-\lambda_{1} \lambda_{3}\right) w_{n+1}\right) w_{n+2}+\left(\lambda_{2}-\lambda_{4}\right)^{2} w_{n+1}^{2}\right) .
\end{aligned}
$$

Explicit forms for $A_{i j}\left(w_{n+1}, w_{n+2}\right)$ associated with fourth order difference equation (3.17):

$$
\begin{aligned}
& A_{11}=0, \\
& \begin{aligned}
A_{12} & =\alpha_{11} \lambda_{1}\left(\lambda_{1} w_{n+1}+\lambda_{3}\right), \\
A_{13} & =\lambda_{1}\left(\lambda_{1} w_{n+1}+\lambda_{3}\right)\left(\alpha_{11}\left(w_{n+1}+w_{n+2}\right)+\alpha_{12}\right), \\
A_{22} & =\alpha_{12} \lambda_{1}\left(\lambda_{1}\left(w_{n+1}+w_{n+2}\right)+2 \lambda_{3}\right)+\alpha_{11}\left(\lambda _ { 1 } \left(\lambda_{1}\left(w_{n+1}^{2}+w_{n+2}^{2}\right)\right.\right. \\
& \left.\left.+\left(3 \lambda_{1} w_{n+1}+4 \lambda_{3}\right) w_{n+2}+4 \lambda_{3} w_{n+1}+\lambda_{4}\right)+\lambda_{3}\left(3 \lambda_{3}-\lambda_{2}\right)\right),
\end{aligned}
\end{aligned}
$$




$$
\begin{aligned}
A_{23} & =\alpha_{12} \lambda_{1}\left(\left(\lambda_{1} w_{n+1}+2 \lambda_{3}\right) w_{n+2}+\left(\lambda_{1} w_{n+1}+\lambda_{2}\right) w_{n+1}+\lambda_{4}\right) \\
& +\alpha_{11}\left(\lambda _ { 1 } \left(\left(3 \lambda_{3}+2 \lambda_{1} w_{n+1}\right) w_{n+2}^{2}+\left(3 \lambda_{1} w_{n+1}^{2}+2 \lambda_{2} w_{n+1}+\lambda_{4}\right) w_{n+2}\right.\right. \\
& \left.\left.+\lambda_{1} w_{n+1}^{3}+\lambda_{2} w_{n+1}^{2}+\lambda_{4} w_{n+1}\right)+\lambda_{3}\left(\lambda_{2}-3 \lambda_{3}\right) w_{n+2}\right), \\
A_{33} & =\alpha_{12} \lambda_{1}\left(\lambda_{3} w_{n+2}^{2}+\left(2 \lambda_{3} w_{n+1}+\lambda_{4}\right) w_{n+2}+\left(\lambda_{3} w_{n+1}+\lambda_{4}\right) w_{n+1}\right)+\alpha_{11}\left(\lambda _ { 1 } \left(\lambda_{1} w_{n+1}\right.\right. \\
& \left.+2 \lambda_{3}\right) w_{n+2}^{3}+\left(2 \lambda_{1}\left(\lambda_{1} w_{n+1}+\lambda_{2}\right) w_{n+1}+\lambda_{1} \lambda_{4}+2 \lambda_{3}\left(\lambda_{2}-3 \lambda_{3}\right)\right) w_{n+2}^{2}+\left(\lambda_{1}^{2} w_{n+1}^{3}\right. \\
& \left.+2 \lambda_{1} \lambda_{2} w_{n+1}^{2}+\left(\lambda_{2}^{2}+2 \lambda_{1} \lambda_{4}-2 \lambda_{2} \lambda_{3}-3 \lambda_{3}^{2}\right) w_{n+1}+\lambda_{4}\left(\lambda_{2}-3 \lambda_{3}\right)\right) w_{n+2} \\
& \left.+2 \lambda_{1} \lambda_{3} w_{n+1}^{3}+\left(\lambda_{1} \lambda_{4}+2 \lambda_{2} \lambda_{3}-6 \lambda_{3}^{2}\right) w_{n+1}^{2}+\lambda_{4}\left(\lambda_{2}-3 \lambda_{3}\right) w_{n+1}\right) .
\end{aligned}
$$

Explicit forms for $A_{i j}\left(w_{n+1}, w_{n+2}\right)$ associated with fourth order difference equation (3.18):

$$
\begin{aligned}
A_{11}= & \alpha_{8} \lambda_{1}^{2}, \\
A_{12}= & \lambda_{1}\left(\alpha_{8}\left(4 \lambda_{1} w_{n+1}+2 \lambda_{1} w_{n+2}+\lambda_{3}\right)+2 \alpha_{12} \lambda_{1}\right), \\
A_{13}= & \lambda_{1}\left(2 \lambda_{1} w_{n+1}+\lambda_{1} w_{n+2}+\lambda_{3}\right)\left(2 \alpha_{8} w_{n+1}+\alpha_{8} w_{n+2}+2 \alpha_{12}\right), \\
A_{22}= & \alpha_{12}\left(2 \lambda_{1} \lambda_{2}+8 \lambda_{1}^{2}\left(w_{n+2}+w_{n+1}\right)\right)+\alpha_{8}\left(2 \lambda_{1} \lambda_{2}\left(w_{n+1}+w_{n+2}\right)\right. \\
& \left.+2 \lambda_{1}^{2}\left(8 w_{n+2} w_{n+1}+3\left(w_{n+1}^{2}+w_{n+2}^{2}\right)\right)+\lambda_{1} \lambda_{4}+2 \lambda_{3}^{2}-\lambda_{2} \lambda_{3}\right), \\
A_{23} & =\alpha_{12}\left(2 \lambda_{1}^{2}\left(2 w_{n+1}^{2}+4 w_{n+2} w_{n+1}+w_{n+2}^{2}\right)+2 \lambda_{1} \lambda_{2} w_{n+2}+4 \lambda_{1}\left(\lambda_{2}-\lambda_{3}\right) w_{n+1}\right. \\
& \left.+2 \lambda_{1} \lambda_{4}\right)+\alpha_{8}\left(2 \lambda_{1} \lambda_{2} w_{n+2}^{2}+2 \lambda_{1}^{2}\left(2 w_{n+1}^{3}+w_{n+2}^{3}+7 w_{n+2} w_{n+1}^{2}+5 w_{n+2}^{2} w_{n+1}\right)\right. \\
& +6 \lambda_{1} \lambda_{2} w_{n+1} w_{n+2}-\lambda_{1} \lambda_{3}\left(4 w_{n+1}^{2}+w_{n+2}^{2}+4 w_{n+2} w_{n+1}\right)+\lambda_{1} \lambda_{4}\left(w_{n+2}+2 w_{n+1}\right) \\
& \left.+\lambda_{2} \lambda_{3} w_{n+2}-2 \lambda_{3}^{2} w_{n+2}+4 \lambda_{1} \lambda_{2} w_{n+1}^{2}\right), \\
A_{33}= & \alpha_{12}\left(2 \lambda_{1} \lambda_{4}\left(w_{n+1}+w_{n+2}\right)+2 \lambda_{1} \lambda_{2} w_{n+1} w_{n+2}+2 \lambda_{1} \lambda_{3}\left(w_{n+1}^{2}+w_{n+2}^{2}\right)\right. \\
& \left.+2 \lambda_{1}^{2} w_{n+1} w_{n+2}\left(w_{n+1}+w_{n+2}\right)\right)+\alpha_{8}\left(-2 \lambda_{2} \lambda_{3} w_{n+1}^{2}+2 \lambda_{3}^{2} w_{n+2} w_{n+1}\right. \\
& +2 \lambda_{2}^{2} w_{n+2} w_{n+1}-2 \lambda_{2} \lambda_{3} w_{n+2}^{2}+\lambda_{1}^{2} w_{n+2}^{4}+\lambda_{1}^{2} w_{n+1}^{4}-2 \lambda_{4} \lambda_{3} w_{n+1}+\lambda_{1} \lambda_{4} w_{n+2}^{2} \\
& +11 \lambda_{1}^{2} w_{n+1}^{2} w_{n+2}^{2}+8 \lambda_{1} \lambda_{2} w_{n+1} w_{n+2}^{2}-9 \lambda_{1} \lambda_{3} w_{n+1} w_{n+2}^{2}+8 \lambda_{1} \lambda_{2} w_{n+1}^{2} w_{n+2} \\
& -9 \lambda_{1} \lambda_{3} w_{n+1}^{2} w_{n+2}+3 \lambda_{1} \lambda_{4} w_{n+1} w_{n+2}-5 \lambda_{2} \lambda_{3} w_{n+2} w_{n+1}+6 \lambda_{1}^{2} w_{n+2}^{3} w_{n+1} \\
& +2 \lambda_{1} \lambda_{2} w_{n+2}^{3}-2 \lambda_{1} \lambda_{3} w_{n+2}^{3}+6 \lambda_{1}^{2} w_{n+1}^{3} w_{n+2}+\lambda_{4} \lambda_{2} w_{n+2}-2 \lambda_{3} \lambda_{4} w_{n+2} \\
& \left.+2 \lambda_{1} \lambda_{2} w_{n+1}^{3}-2 \lambda_{1} \lambda_{3} w_{n+1}^{3}+\lambda_{1} \lambda_{4} w_{n+1}^{2}+\lambda_{4} \lambda_{2} w_{n+1}+\lambda_{2}^{2} w_{n+2}^{2}+\lambda_{2}^{2} w_{n+1}^{2}\right) . \\
& -19 A_{1}\left(w_{n}\right)
\end{aligned}
$$

Explicit forms for $A_{i j}\left(w_{n+1}, w_{n+2}\right)$ associated with fourth order difference equation (3.19):

$$
\begin{aligned}
& A_{11}=A_{12}=0, \\
& A_{13}=\left(\lambda_{1} w_{n+1}+\lambda_{4}+\lambda_{5}\right)\left(\lambda_{4}+\lambda_{1} w_{n+1}-\lambda_{5}\right), \\
& A_{22}=\left(\lambda_{1} w_{n+1}+\lambda_{4}+\lambda_{5}\right)\left(\lambda_{1} w_{n+2}+\lambda_{4}+\lambda_{5}\right), \\
& A_{23}=\left(\lambda_{1} w_{n+1}+\lambda_{4}+\lambda_{5}\right)\left(2 \lambda_{4} w_{n+1}+\lambda_{5} w_{n+2}+\lambda_{4} w_{n+2}+\lambda_{3}\right),
\end{aligned}
$$




$$
\begin{aligned}
A_{33} & =\left(\lambda_{4}+\lambda_{5}\right)\left(\left(\lambda_{4}+\lambda_{5}\right) w_{n+2} w_{n+1}+\lambda_{3}\left(w_{n+1}+w_{n+2}\right)+\lambda_{4}\left(w_{n+2}^{2}+w_{n+1}^{2}\right)\right. \\
& \left.-\lambda_{5}\left(w_{n+2}^{2}+w_{n+1}^{2}\right)\right) .
\end{aligned}
$$

\section{Appendix B}

Consider an Nth-order autonomous $O \Delta E$ having the form

$$
w_{n+N}=F\left(w_{n}, \ldots w_{n+N-1}\right) .
$$

Let us assume that equation (B1) admits a polynomial integral $I(n)$ having the form

$$
I(n)=\sum_{j=1}^{3}\left[A_{1 j}(n) w_{n+N-1}^{2}+A_{2 j}(n) w_{n+N-1}+A_{3 j}(n)\right] w_{n}^{3-j},
$$

where

$$
A_{i j}(n)=A_{i j}\left(w_{n+1}, w_{n+2}, \ldots, w_{n+N-2}\right), i, j=1,2,3
$$

are unknown functions. Then the integrability condition $I(n+1)-I(n)=0$ leads to a quadratic equation in $w_{n+N}$

$$
F_{1}(n) w_{n+N}^{2}+F_{2}(n) w_{n+N}+F_{3}(n)-\left(F_{4}(n) w_{n}^{2}+F_{5}(n) w_{n}+F_{6}(n)\right)=0,
$$

where

$F_{i}(n)=\sum_{j=1}^{3} A_{i j}(n+1) w_{n+1}^{3-j}, i=1,2,3, F_{k}(n)=\sum_{j=1}^{3} A_{j k}(n) w_{n+N-1}^{3-j}, \quad k=1,2,3$.

It is straightforward to check that equation (B3) can be factorised as

$$
\left(w_{n+N}+w_{n}+\frac{F_{2}(n)+F_{5}(n)}{2 F_{1}(n)}\right)\left(w_{n+N}-w_{n}+\frac{F_{2}(n)-F_{5}(n)}{2 F_{1}(n)}\right)=0
$$

provided

$A_{11}(n+1) w_{n+1}^{2}-A_{11}(n) w_{n+N-1}^{2}+A_{12}(n+1) w_{n+1}-A_{21}(n) w_{n+N-1}=A_{31}(n)-A_{13}(n+1)(B 5 a)$

and

$$
\left[F_{5}(n)+F_{2}(n)\right]\left[F_{5}(n)-F_{2}(n)\right]=4\left[F_{6}(n)-F_{3}(n)\right] F_{1}(n) .
$$

Thus we obtain,

$$
\begin{aligned}
& w_{n+N}=-w_{n}-\frac{F_{2}(n)+F_{5}(n)}{2 F_{1}(n)}, \\
& w_{n+N}=w_{n}-\frac{F_{2}(n)-F_{5}(n)}{2 F_{1}(n)} .
\end{aligned}
$$

Obviously (B5b) is satisfied for distinct possibilities

(1) $F_{5}=F_{2}$ and $F_{6}=F_{3}$, 
(2) $F_{5} \neq F_{2}$ and $F_{6} \neq F_{3}$.

Demanding that one of the integrals of the given $O \Delta E$ (B1), say, $I_{1}(n)$ is cyclic invariant, that is

$$
\begin{aligned}
& I_{1}\left(w_{n}, w_{n+1}, \cdots, w_{n+N-2}, w_{n+N-1}\right)=I_{1}\left(w_{n+1}, w_{n+2}, \cdots, w_{n+N-1}, w_{n}\right) \\
& \quad=I_{1}\left(w_{n+2}, w_{n+3}, \cdots, w_{n}, w_{n+1}\right)=\cdots=I_{1}\left(w_{n+N-1}, w_{n}, \cdots, w_{n+N-3}, w_{n+N-2}\right)
\end{aligned}
$$

it is easy to verify that both the conditions given in equation (B5a) as well as $F_{5}=F_{2}$ and $F_{6}=F_{3}$ satisfy. In this case the integral $I_{1}$ will take the form

$$
I_{1}(n)=\sum_{0 \leq i_{1} \leq i_{2} \leq \ldots \leq i_{n} \leq 2} a_{i_{1} i_{2} \ldots i_{n}} w_{n+1}^{i_{1}} w_{n+2}^{i_{2}} \ldots w_{n+N+1}^{i_{n}},
$$

where

$$
a_{i_{1}, i_{2}, \cdots, i_{n-1}, i_{n}}=a_{i_{2}, i_{3}, \cdots, i_{n}, i_{1}}=\cdots=a_{i_{n}, i_{1}, \cdots, i_{n-2}, i_{n-1}} \text {, provided } i_{1} \neq i_{2} \neq \cdots \neq i_{n} .
$$

Remaining integrals $I_{2}(n), I_{3}(n) \cdots I_{N-1}(n)$ may be constructed by demanding them not to be cyclic invariant. We find that the integrals (2.24) of the third order difference equation (2.22) and (2.27) of (2.25) are cyclic invariant.

\section{References}

[1] Ablowitz M J and Ladik J F, A nonlinear difference scheme and inverse scattering, Stud. Appl. Math. 55 (1976), 213-229.

[2] Ablowitz M J and LAdiK J F, Nonlinear differential-difference equations and Fourier analysis. J. Math. Phys. 17 (1976), 1011-1018.

[3] Arnold V I, Mathematical methods of classical mechanics, Springer-Verlag, Berlin 1978.

[4] Bellon M and Viallet C M ,Algebraic entropy. Commun. Math. Phys. 204 (1999), 425437.

[5] Bruschi M, Ragnisco O, Santini P M and Gui zhang tu, Integrable symplectic maps. Physica D. 49 (1991), 273-294.

[6] Byrnes G B, Haggar F and Quispel G R W, Sufficient conditions for dynamical systems to have pre-symplectic or pre-implectic structures. Physica A. 272 (1999) 99-129.

[7] Capel H W, Nijhoff F W and Papageorgiou V G, Complete integrability of Lagrangian mappings and lattices of K-dV type. Phys. Lett. A. 155 (1991), 377 - 387.

[8] Capel H W and Sahadevan R, A new family of four dimensional symplectic and integrable mappings. Physica A. 289 (2001), 86-106.

[9] Discrete Integrable Systems, Editors: Grammaticos B,Kosmann-Schwarzbach Y and Tamizhmani T, Springer-Verlag, Berlin, 2004.

[10] Grammaticos B, Ramani A and Papageorgiou V, Do integrable mappings have the Painleve property?, Phys. Rev. Lett. 67 (1991), 1825-1828. 
[11] Halburd R G, Diophantine integrability J. Phys. A: Math. Gen. 38 (2005), L1-L7.

[12] Hietarinta J and Viallet C, Singularity confinement and chaos in discrete systems. Phys. Rev. Lett. 81 (1998), 325-328.

[13] Hydon P E, Conservation laws of partial difference equations with two independent variables. J. Phys. A: Math. Gen. 34 (2001), 10347-10355.

[14] IAtrou A, Three dimensional integrable mappings. arxiv:nlin 0306052v1 (2003).

[15] Iatrou A, Higher dimensional integrable mappings. Physica D. 179 (2003), 101-125.

[16] Lafortune S and Goriely A , Singularity confinement and algebraic integrability. J.Math.Phys. 45 (2004), 1191-1208.

[17] Quispel G R W, Capel H W, Papageorgiou V G and Nijhoff F W, Integrable mappings derived from soliton equations. Physica A. 173 (1991), 243-266.

[18] Quispel G R W, Capel H W and Roberts J A G, Duality for discrete integrable systems. J.Phys. A: Math. and Gen. 38 (2005), 3965 - 3980.

[19] Quispel G R W, Roberts J A G and Thompson C J, Integrable mappings and soliton equations. Phys.Lett. A. 126 (1988), 419 - 421.

[20] Quispel G R W, Roberts J A G and Thompson C J, Integrable mappings and soliton equations II. Physica D. 34 (1989), 183 - 192.

[21] Roberts J A G and Quispel G R W Creating and relating 3-dimensional integrable maps. J.Phys.A: Math. Gen. 39 (2006), L605-L615.

[22] RuijsenaArs S, First order analytic difference equations and integrable quantum systems. J.Math. Phys. 38 (1997), 1069-1146.

[23] Sahadevan R, Uma Maheswari C, Direct method to construct integrals for Nth- order autonomous ordinary difference equations. Proc. Roy. Soc. A. 464 (2008), 341-364.

[24] Tremblay S, Grammaticos B and Ramani A, Integrable lattice equations and their growth properties. Phys.Lett. A. 278 (2001), 319-324.

[25] Veselov A P Integrable maps. Russian Math. Surveys. 46 (1991), 1-51. 